\title{
Band Coverage in Wireless Sensor Networks: A Preliminary Study
}

\author{
Yi-hui LI ${ }^{1, a}$ and Gao-xi XIAO ${ }^{2, b,{ }^{*}}$ \\ ${ }^{1}$ Panasonic R\&D Center Singapore \\ ${ }^{2}$ School of Electrical and Electronic Engineering, Nanyang Technological University, \\ Singapore \\ ayihui.li@sg.panasonic.com, begxxiao@ntu.edu.sg \\ ${ }^{*}$ Corresponding author
}

Keywords: Wireless sensor networks, Sleep-wakeup scheme, Band coverage.

\begin{abstract}
In this letter, we propose a novel sleep-wakeup mechanism, named band coverage scheme, for wireless sensor networks, with the objective of achieving high energy efficiency and high successful rate of event/intrusion detections in an area with a relatively large width. Unlike the classic barrier coverage, which typically adopts a certain type of randomized sleep-wakeup algorithm, the band coverage scheme divides the monitored region into a number of narrow bands and makes the sensors inside different bands sleep and wakeup in sequence. Preliminary results show that, band coverage has merits in saving energy consumption and easing information integration while ensuring a high successful rate in intrusion detection.
\end{abstract}

\section{Introduction}

In the past decades, wireless sensor networks (WSNs) have gained wide deployments for various military and civil applications. In many of these applications, the task of the WSN is to monitor a certain region, detecting specific types of events (e.g., forest fire) or intrusion in the region. A WSN is typically composed of a large number of small-size, inexpensive and low-power sensor nodes (Hereafter, we use the words sensor, sensor nodes and nodes interchangeably.) with data collection and dissemination functions [1]. Each sensor may respond to only a single type of physical stimulus (e.g., heat, light, sound, pressure, etc.). It converts the quantity or parameter of this stimulus into recordable signals [2]. Different types of sensors provide different types of sensing information which, when being integrated together, help achieve reliable detection of event/intrusion.

A critical constraint imposing on most WSNs is the limited energy resources they have. Sensors are usually powered by small batteries with limited energy supplies which cannot be conveniently replaced or recharged. To improve the energy efficiency of WSNs in different scenarios, an enormous number of different schemes have been proposed, with different main ideas of forming up sensor clusters [3-5], using energy harvesting sensors [6-8], and many others. Among these results, the ones most closely related to our work to be reported in this letter are those on sleep-wakeup schemes, where sensors switch between sleep and wakeup modes (e.g., [9-13]). Sensors are functioning (namely, sensing environment and transmitting information) only in the wakeup mode; while in the sleep 
mode, their energy consumptions are minimized. By properly scheduling the sleep and wakeup time, a WSN's energy consumptions can be reduced while still ensuring a high successful rate in fulfilling sensing tasks.

Among the vast existing sleep-wakeup schemes, barrier coverage schemes stand out as probably the most favorable option for intrusion detection in a belt area [11-13]. The main idea is typically to carefully control the probabilities for each sensor to be in sleep and wakeup modes respectively, such that any intruder going across the belt area has a guaranteed or almost guaranteed chance of triggering a certain number of alarms.

While barrier coverage works efficiently for a belt area with a relatively narrow width, it may not be an equally favorable option for monitoring a wider area, e.g., an area closely resembling a large rectangle. One reason is the relatively large number of sensors that have to be active at any moment to ensure reliable event/intrusion detection. It is worth the efforts to study how to reduce the number of active sensors without sacrificing the reliability of detection function.

Another reason why barrier coverage may not work so well in a wider area is that it may not conveniently support information integration, i.e., the integration and correlation of different information sensed by different types of sensors, which may be of crucial importance for some applications. For example, combined sensing of wind, rain and ground vibration may help lower the false alarm rate in intrusion detection; and sound and vibration information may be correlated to help figure out the type of intruder(s). To achieve simple yet reliable information integration and correlation, different types of sensors at wakeup state may have to be reasonably close to each other around the location when an event/intruder is detected: two alarm signals sent out by two sensors relatively far away from each other may be indeed triggered by the same intruder or two totally irrelevant events.

In this letter, we propose a novel band coverage scheme for energy efficiency event/intrusion detection in a relatively wide area. The main idea is to divide the region to be sensed into a number of relatively narrow bands and let the sensors in each band be in wakeup state in a sequence. By doing so, we ensure that (i) the sensors only need a short communication/detection range to achieve reliable event detection, which helps prolong the network lifetime; and (ii) sensors in the wakeup state can easily keep a reasonably high density to allow relatively easier information integration and correlation.

The rest of this letter is organized as follows. We present necessary definitions and network model and then propose the band coverage scheme in Section II. Preliminary simulation results and discussions are presented in Section III. And finally, Section IV concludes the letter.

\section{Band Coverage Scheme}

We start by considering a large rectangular region with a number of sensors randomly distributed at a reasonably high density, high enough to enable the system fulfill the sensing and information integration functions. Without loss of generality, we adopt a disc-based sensing model, where any object within the sensing range $r$ of an active sensor can be reliably detected. An example case of the proposed system is illustrated in Fig. 1. Specifically, we assume that there are two different types of sensors in this network: 
- Regular sensor nodes (denoted as “ $\bigcirc$ " in Fig. 1), which are equipped with necessary sensing/transmission functions and are capable of carrying out sleep/wakeup operations. As that in many other WSNs adopting the typical sleep/wakeup schemes, the regular sensor nodes can be activated into wakeup mode by wakeup signals sent by nearby sensors; some or all sensors may also have internal clocks (to be discussed in detail later), with no need of global synchronization, to allow periodical sleep/wakeup operations.

- Stimulus sensor nodes (denoted as " $\square$ " in Fig. 1), which are nearly the same as the regular sensors. The only additional function they have is that they can be "activated" into wakeup mode by a special activating signal from the base station. The regular sensor nodes will ignore the special activating signal from the base station.

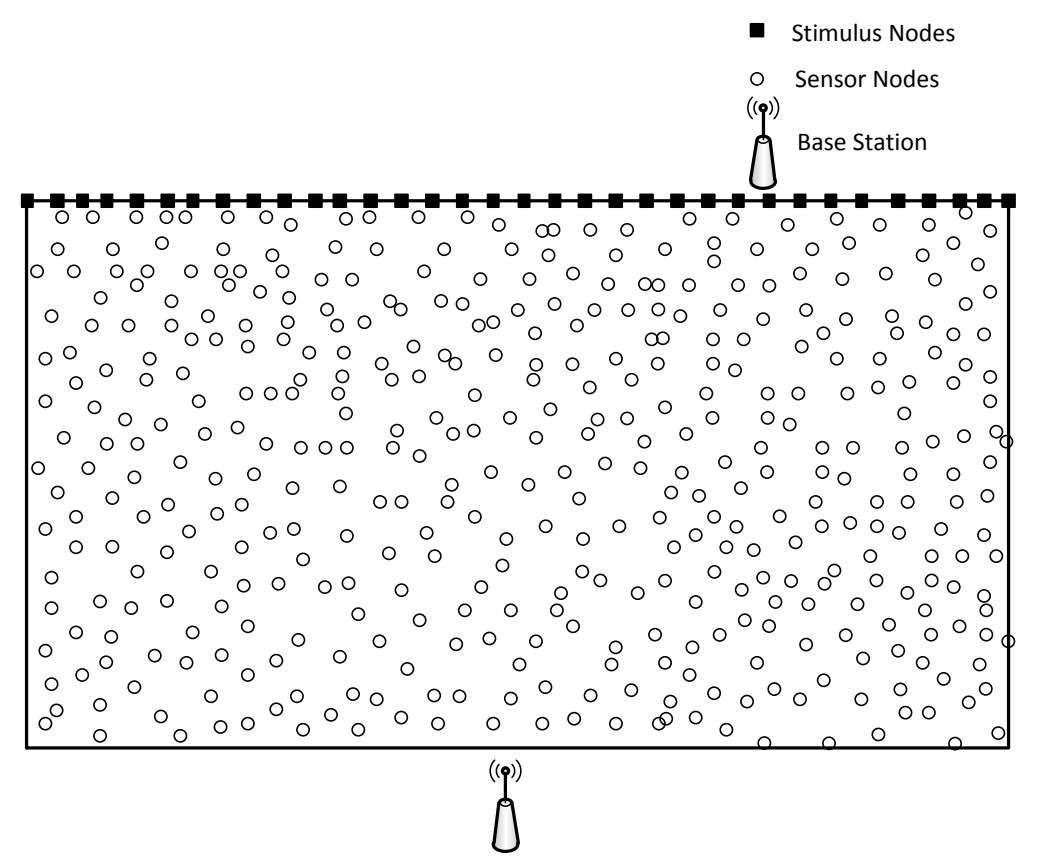

Figure 1: Components of a wireless sensor network for band coverage.

As illustrated in Fig.1, the stimulus sensors are distributed along the edge of the region relatively far away from the base station.

The main idea is to divide the monitored region into a certain number of bands. At the beginning, the stimulus sensors are activated by the base station. The activation may use a certain activating signal which makes sense only to the stimulus sensors. Each stimulus sensors, upon receiving the activating signal, will send out a wakeup signal. With the limited communication range of the stimulus sensors, only those sensors within a narrow band region close to the stimulus sensors will be waken up (as illustrated in Fig. 2(a)). The sensors in this narrow band shall monitor their respective sensing regions. If, and only if, any event is detected, an alarm signal will be sent out. After a certain short period of time (termed as active period of each band), the sensors in this narrow band will send a wakeup signal to activate the sensors in the next band region, as illustrated in Fig. 2(b).

Sensors in the second narrow band region will carry out their monitoring job and at the end of their active period, send wakeup signals to wake up the sensors in the third band 
region. The sensors in the first narrow band region meanwhile will ignore this wakeup signal as they just finished their work in the last iteration. The procedure is repeated until the wakeup signals sent by the last narrow band region reach the base station. The base station, upon receiving such wakeup signals, will send an activating signal to the stimulus sensors again. Stimulus sensors will keep record of the time interval between the two activating signals. And will send out further wakeup signal with the same time interval. The base station therefore does not need to send out further activating signals; such may be of values in some applications, e.g., while base station should keep silence most of time to lower the chance of revealing its own location. However, if the base station does choose to send further activating signals, say, in order to re-synchronize the operations of all the sensors in each band area, the stimulus sensors will still send out wake-up signal immediately upon receiving the activating signal.

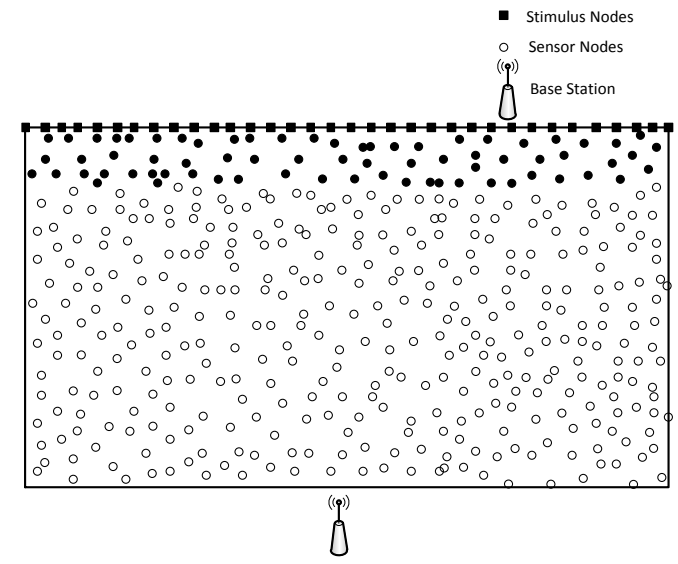

(a)

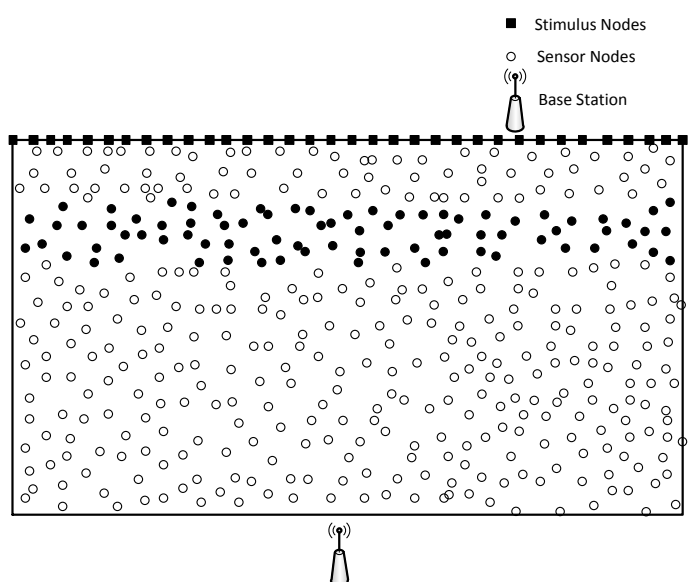

(b)

Figure 2: An illustration of the band coverage scheme in the first two iterations: (a) sensors in the first band region get activated by the stimulus sensors; (b) sensors in the second band region get activated by sensors in the first band region.

If good synchronization is not of a critical concern and all the sensors have internal clocks, it is also possible to let all the sensors keep record of the time interval and wakeup periodically at that time interval. Certainly, combined schemes can also be designed, e.g., to let every sensor have periodical sleep-wakeup following their own internal clocks for a certain period of time, before switching back to allow being waken up by wakeup signals.

\section{Preliminary Results and Discussions}

As the first step of a preliminary study, we compare band coverage vs. barrier coverage in the number of alarms that can be triggered with roughly the same number of active sensors in each time slot. Further comparisons between energy consumptions, lifespan and easiness of information integration will be reported in our future work.

Assume that the deployment region is a $300 \mathrm{~m} \times 300 \mathrm{~m}$ rectangular region, the sensing radium is $15 \mathrm{~m}$ and communication radium is $30 \mathrm{~m}$. Rough calculations show that in this way, a band region would be a $30 \mathrm{~m} \times 300 \mathrm{~m}$ rectangular region. For barrier coverage, we also let every sensor be active at a probability of $10 \%$ in each time interval. Figure 3 
compares the simulation results showing that the minimum number of alarms an intruder going across the whole region would trigger. Figure 3(a) illustrates the average results of 10 independent uniform random sensor distributions with 50 realizations for each distribution. Figure 3(b) presents the worst-case results among the 50 realizations for each of the 10 distributions. As we can see, with roughly the same number of sensors being active in each time slot, band coverage steadily achieves a higher number of alarms for a going-across intruder, ensuring a more reliable monitoring and detection of intrusion. In other words, to achieve the same reliability level of event/intrusion detection, band coverage scheme requests a smaller sensing/communication radium of sensors or a smaller average number of active sensors at any moment, which helps save energy and prolong network lifespan

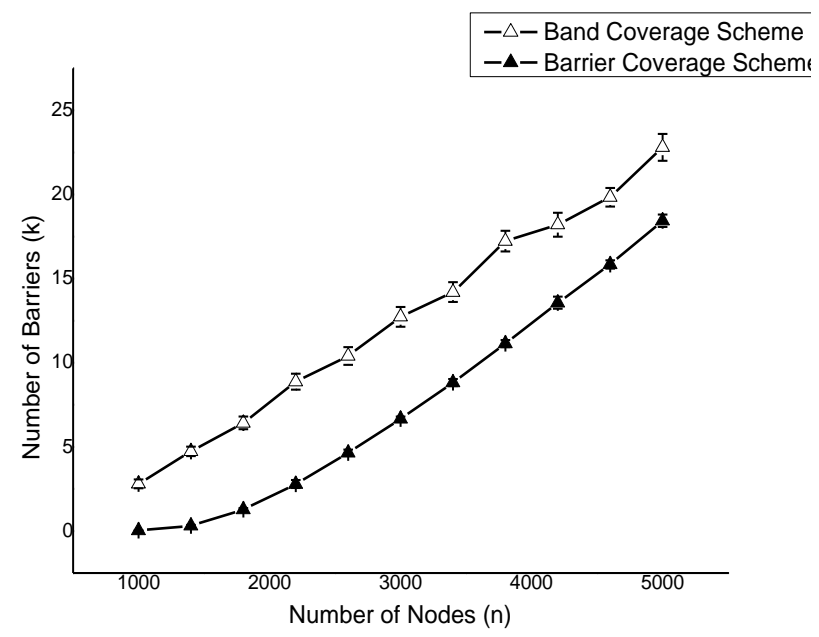

(a)

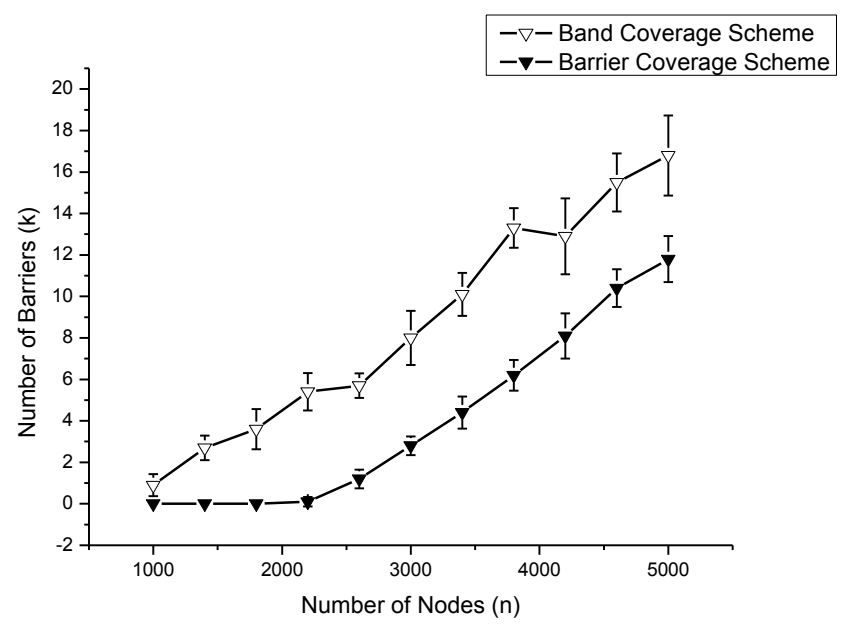

(b)

Figure 3: Comparison between band coverage and barrier coverage in the number of alarms to be triggered by an intruder: (a) average results; (b) the worst-case results. 
As all the active sensors groom together in a narrow band region, intuitively we may expect that a lower energy consumption when inter-sensor communications are needed, and easier information integration as well. Careful further studies however are needed for comprehensive evaluation, and further improvements as well.

\section{Summary}

In this letter, we proposed a novel band coverage scheme for wireless sensor networks, with the objective of achieving higher energy efficiency and high successful rate of event/intrusion detections in an area with a relatively large width. The main idea is to divide the monitored region into a number of bands and make the sensors inside different bands sleep and wakeup in sequence. Preliminary results show that band coverage has merits in saving energy consumption while ensuring a high successful rate in event/intrusion detection. Further studies however are needed to further improve the proposed scheme and verify its favorite features and satisfactory performance.

\section{Acknowledgement}

This research is partially supported by Ministry of Education, Singapore, under contract of RG28/14.

\section{References}

[1] I. F. Akyildiz, S. Weilian, Y. Sankarasubramaniam, and E. Cayirci, "A survey on sensor networks," Communications Magazine, IEEE, vol. 40, pp. 102-114, 2002.

[2] J. Wilson, Sensor Technology Handbook. CA, USA: Elsevier, 2005.

[3] W. R. Heinzelman, A. Chandrakasan, and H. Balakrishnan, "Energy efficient communication protocol for wireless microsensor networks," Proc. Hawaii Int. Conf. Syst. Sci. 2000.

[4] D. Mahmood, N. Javaid, S. Mahmood, S. Qureshi, A. M. Memon, and T. Zaman, "MOELEACH: a variant of LEACH for WSNs," arxiv: 1307.7059v1, 2013.

[5] Y. Li, G. Xiao, G. Singh, and R. Gupta, "Algorithms for finding best locations of cluster heads for minimizing energy consumption in wireless sensor networks," Wireless Networks, vol. 19, no. 7, pp. 1755-1768, Oct. 2013.

[6] A. Kansal, J. Hsu, S. Zahedi, and M. B. Srivastava, "Power management in energy harvesting sensor networks," ACM Trans. Embeded Comput. Systs., vol. 6, no. 4, Sept. 2007.

[7] S Sudevalayam and P Kulkarni, "Energy Harvesting Sensor Nodes: Survey and Implications,” IEEE Commun. Surveys \& Tutor., vol. 13, no. 3, pp. 443-461, 2011.

[8] P. Zhang, G. Xiao, and H.-P. Tan, "Clustering Algorithms for Maximizing the Lifetime of Wireless Sensor Networks with Energy-Harvesting Sensors," Computer Networks, vol. 57, no. 14, pp. 2689-2704, Oct. 2013. 
[9] A. Keshavarzian, H. Lee, and L. Venkatraman, "Wakeup scheduling in wireless sensor networks," Proc. MobiHoc'06, May 2006.

[10] G. Anastasi, M. Conti, M. Di Francesco, "Extending the lifetime of wireless sensor networks through adaptive sleep," IEEE Trans. Industrial Informatics, vol. 5, no. 3, pp. 351-365, Aug. 2009.

[11] S. Kumar, T. H. Lai, and A. Arora, "Barrier coverage with wireless sensors," in Proc. MobiCom, Cologne, Germany, 2005, pp. 284-298.

[12] S. Kumar, T. H. Lai, and J. Balogh, "On k-coverage in a mostly sleeping sensor network," Wireless Networks, vol. 14, pp. 277-294, 2008.

[13] J.-E. Kim, M.-K. Yoon, J. Han, and C.-G. Lee, "Sensor placement for 3-coverage with minimum separation requirements," in Proc. DCOSS, Santorini, Greece, 2008, pp. 266-281. 